\title{
Capsule Commentary on Ratelle et al., Predicting and Communicating Risk of Clinical Deterioration: An Observational Cohort Study of Internal Medicine Residents
}

\author{
Jennifer C. Thompson, MD, FACP \\ J Gen Intern Med 30(4):492 \\ DOI: $10.1007 / \mathrm{s} 11606-014-3132-2$ \\ (c) Society of General Internal Medicine 2015
}

Orlando Veterans Affairs Medical Center, Orlando, FL, USA.

T he study by Ratelle et al. ${ }^{1}$ evaluated the ability of Internal I Medicine residents to use a clinical prediction tool, the Patient Acuity Rating (PAR), ${ }^{2}$ to identify patients at risk for clinical deterioration. By evaluating the level of agreement between independently derived PAR scores of residents participating in a patient handoff, the study also investigated how effectively the residents communicated the potential for clinical deterioration. The authors found that higher PAR scores were associated with a significant increase in the odds of clinical deterioration, which is consistent with the findings of the original PAR study. ${ }^{2}$ Among post-graduate year (PGY) 1 residents, there was fair agreement in PAR scores between the giving and the receiving resident after a handoff. Interestingly, the level of agreement of PAR scores was lower among PGY3 residents, with higher average PAR scores by residents receiving the handoff.

As the authors note, a thought-provoking and potentially confounding factor is the impact of formulating the PAR on the subsequent clinical decision-making by the resident receiving the handoff. A benefit of the PAR is that it facilitates the process of synthesizing clinical judgment into a simple, single number. ${ }^{3}$ Since the receiving resident was responsible for the subsequent care of the patient, it is possible that the process of formulating the PAR may have influenced the clinical assessment and subsequent clinical decisions (e.g., the need for escalation of care) beyond the information communicated in the handoff itself.

The study supports a potential role for the PAR in the implementation or assessment of standardized handoff procedures. As an important component of the Accreditation Council for Graduation Medical Education (ACGME) CLER (Clinical
Learning Environment Review) Care Transitions Pathway, ${ }^{4}$ handoffs are subject to increasing study and scrutiny in academic centers. Further investigation is needed to understand the multiple inter-related factors that impact handoff effectiveness, particularly since standardized handoff procedures have recently been associated with a reduction in medical errors and preventable adverse events when implemented in conjunction with communication training and faculty development. ${ }^{5}$

Conflicts of Interest: The author has no conflicts of interest associated with the content of this article.

Disclaimer: The views expressed in this article are those of the author and do not necessarily reflect the position or policy of the Department of Veterans Affairs or the United States government.

Corresponding Author: Jennifer C. Thompson, MD, FACP; Orlando Veterans Affairs Medical Center, Orlando, FL 32803, USA (e-mail: Jennifer.thompson6@va.gov).

\section{REFERENCES}

1. Ratelle JT, Kelm DJ, Halvorsen AJ, West CP, Oxentenko AS. Predicting and communicating risk of clinical deterioration: An observational cohort study of Internal Medicine residents. J Gen Intern Med 2014; doi:10.1007/ s11606-014-3114-4.

2. Edelson DP, Retzer E, Weidman EK, Walsh D, Woodruff J, Cua JL, Schmitz A, Davis AM, Minsky BD, Meadow W, Vanden Hoek TL, Meltzer DO. The patient acuity rating: Quantifying clinical judgment regarding inpatient stability. J Hosp Med. 2011;6(8):475-479.

3. Phillips AW, Yuen TC, Retzer E, Woodruff J, Arora V, Edelsen DP. Supplementing cross-cover communication with the patient acuity rating. $J$ Gen Intern Med. 2012;23(3):406-11.

4. Accreditation Council for Graduate Medical Education. CLER Pathways to Excellence 2014. Available at https://www.acgme.org/acgmeweb/Portals / O/PDFs/CLER/CLER_Brochure.pdf. Accessed November 16, 2014

5. Starmer AJ, Spector ND, Srivastava R, West DC, Rosenbluth G, Allen AD, et al. Changes in medical errors after implementation of a handoff program. N Engl J Med. 2014;371:1803-12. 